\title{
Enhanced Performance of GCE/N-Reduced Graphene Oxide-Au Nanocomposite in Dopamine Sensing ${ }^{\dagger}$
}

\author{
Daria Minta, Piotr Wiench and Grażyna Gryglewicz * \\ Department of Polymer and Carbonaceous Materials, Faculty of Chemistry, Wrocław University of Science \\ and Technology, 50-370 Wrocław, Poland \\ * Correspondence: grazyna.gryglewicz@pwr.edu.pl \\ + Presented at the 7th International Symposium on Sensor Science, Napoli, Italy, 9-11 May 2019.
}

Published: 23 August 2019

\begin{abstract}
The reduced graphene oxide (rGO) and nitrogen-reduced graphene oxide (N-rGO) were electrochemically coated with gold nanoparticles and compared as the modifier of a glassy carbon electrode (GCE) for dopamine (DA) electrochemical sensors. The rGO and N-rGO were prepared by reduction of graphene oxide $(\mathrm{GO})$ under hydrothermal conditions. Urea was applied as a nitrogen dopant. The modified GCEs were tested as working electrodes in the electrochemical DA detection in the presence of ascorbic and uric acids. Initially, the working parameters of sensors in a pure dopamine solution were determined. The GCE/N-rGO-Au electrode exhibited a lower limit of detection compared with the GCE/rGO-Au ( $385 \mathrm{vs.} 700 \mathrm{nM}$ ). Both sensors had a wide linear range of $1-100 \mu \mathrm{M}$ and high sensitivity of $0.78 \mu \mathrm{A} / \mu \mathrm{M}$ for GCE/N-rGO-Au and $1.78 \mu \mathrm{A} / \mu \mathrm{M}$ for GCE/rGO$\mathrm{Au}$. The presence of ascorbic and uric acids in the solution resulted in a decrease in the intensity of DA oxidation peak, maintaining acceptable limit of detection. This study showed that the surface modification of the graphene materials with gold nanoparticles allows to obtain satisfactory working parameters of DA sensors. The GCE/N-rGO-Au demonstrated the superior performance in DA sensing due to a homogeneous distribution of gold nanoparticles on the surface of the Ndoped graphene material.
\end{abstract}

Keywords: dopamine; gold nanoparticles; reduced graphene oxide; nitrogen-doped reduced graphene oxide; electrochemical sensor

\section{Introduction}

Nowadays, great scientific efforts are put into the development of electrode materials in order to obtain sensitive, fast and effective dopamine (DA) detection [1]. Reduced graphene oxide (rGO) and $\mathrm{N}$-doped reduced graphene oxide $(\mathrm{N}-\mathrm{rGO})$ are promising materials in the electrochemical DA detection due to their high electrical conductivity and a high surface to volume ratio $[2,3]$. The introduction of nitrogen atoms into the structure of rGO leads to elimination of oxygen groups and enhancement of electrical conductivity [3]. The main problem of the DA detection is that DA coexists with uric and ascorbic acids in biological samples [4]. Voltammetric responses of these compounds are similar, therefore it is important to use materials which ensure selective DA detection in the presence of different interferents. Increasingly popular, the modification of graphene materials by noble metal nanoparticles, such as gold nanoparticles (AuNPs), results in an enhancement of DA sensing and its selectivity [5]. AuNPs can catalyze red-ox reactions and enhance electron transfer [5]. Deposition of AuNPs on the surface of carbon materials can increase the active surface and enhance the affinity between the electrode material and the analyte such as DA.

In this work, we demonstrate the influence of rGO and N-rGO modified with AuNPs on the electrochemical DA detection. The synthesized graphene materials exhibited different nanoparticles distribution and size ranges on their surfaces. rGO and N-rGO were used for modification of a glassy 
carbon electrode (GCE), which was applied as a working electrode in the DA detection. GCE/N-rGO$\mathrm{Au}$ exhibited a lower limit of DA detection when compared to GCE/rGO-Au (385 vs $700 \mathrm{nM}$ ).

\section{Materials and Methods}

GO was synthesized by the modified Hummers method from the graphite (TimCal). rGO and $\mathrm{N}-\mathrm{rGO}$ were obtained by treatment of $100 \mathrm{ml}$ of GO in an autoclave under hydrothermal conditions at $180{ }^{\circ} \mathrm{C}$ for $5 \mathrm{~h}(\mathrm{rGO})$ and $12 \mathrm{~h}$ in the presence of urea (N-rGO) [6]. A dispersion of the graphene material $(4 \mathrm{mg})$ in a mixture of $1 \mathrm{~mL}$ Mili-Q water and DMF as a dispersion stabilizer (1:1) v/v) was used to modify the GCE by drop-casting. After that, the AuNPs were electrodeposited on the electrode surface from $1 \mathrm{mM} \mathrm{HAuCl}_{4}$ aqueous solution using cyclic voltammetry (CV).

The electrochemical measurements were performed in a three electrode cell using GCE/(N)rGO-Au as a working electrode, a platinum wire as a counter and a saturated calomel electrode as a reference electrode, respectively. The measurements were carried out in a phosphate buffer solution (PBS, pH 7.4) containing $100 \mu \mathrm{M}$ DA as an electrolyte. After each measurement the working electrodes were purified by CV in 7.4 PBS buffer solution to remove DA.

\section{Results and Discussion}

The DA oxidation-reduction reaction was investigated by $\mathrm{CV}$ and differential pulse voltammetry (DPV) which enable to determine the working parameters of the DA sensors (Table 1). As shown on Figure 1, the modification of GCE with N-rGO and N-rGO/Au results in an enhancement of the DA oxidation peak. The oxidation potential for both electrodes was comparable (Table 1). GCE/N-rGO-Au exhibits a lower detection limit and sensitivity when compared with GCE/rGO-Au (385 vs. $700 \mathrm{nM}$ and 0.78 vs. $1.78 \mu \mathrm{A} / \mu \mathrm{M}$, respectively).

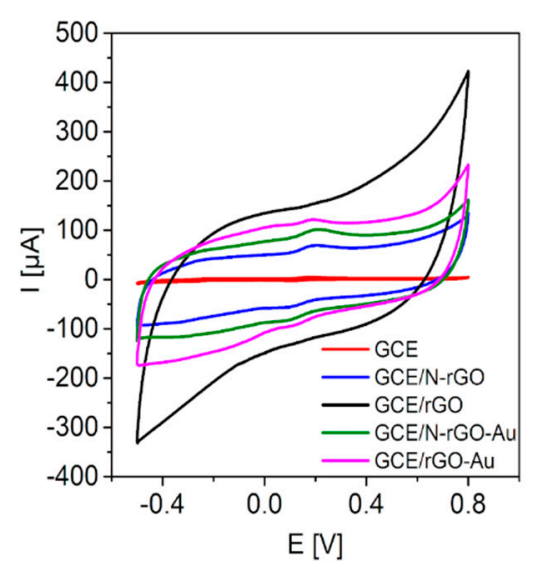

Figure 1. CVs of the examined electrodes in $0.1 \mathrm{M}$ PBS containing $100 \mu \mathrm{M}$ of DA.

Table 1. Working parameters of DA sensors.

\begin{tabular}{ccccc}
\hline $\begin{array}{c}\text { Working } \\
\text { Electrode }\end{array}$ & $\begin{array}{c}\text { LOD, } \\
\mathbf{n M}\end{array}$ & $\begin{array}{c}\text { Linear Range, } \\
\boldsymbol{\mu M}\end{array}$ & $\begin{array}{c}\text { Sensitivity, } \\
\boldsymbol{\mu A} / \boldsymbol{\mu} \mathbf{M}\end{array}$ & $\begin{array}{c}\text { Oxidation Potential } \\
\mathbf{m V}\end{array}$ \\
\hline GCE/N-rGO-Au & 385 & $1-100$ & 0.78 & 210 \\
GCE/rGO-Au & 700 & $1-100$ & 1.78 & 190 \\
GCE & 5000 & $10-100$ & 0.12 & 190 \\
\hline
\end{tabular}

The morphology of the graphene materials was examined by the field emission-scanning electron microscopy (FESEM) (Figure 2). N-rGO-Au exhibited a high concentration and homogenous distribution of AuNPs on its entire surface, despite fewer cycles of electrodeposition than for rGO (20 vs. 30 cycles). Moreover, the Au nanoparticles deposited on the N-rGO were smaller than those of rGO-Au (20-260 nm vs. 30-450 nm). This suggests that AuNPs are preferentially deposited on the Nrich regions of the graphene material. 


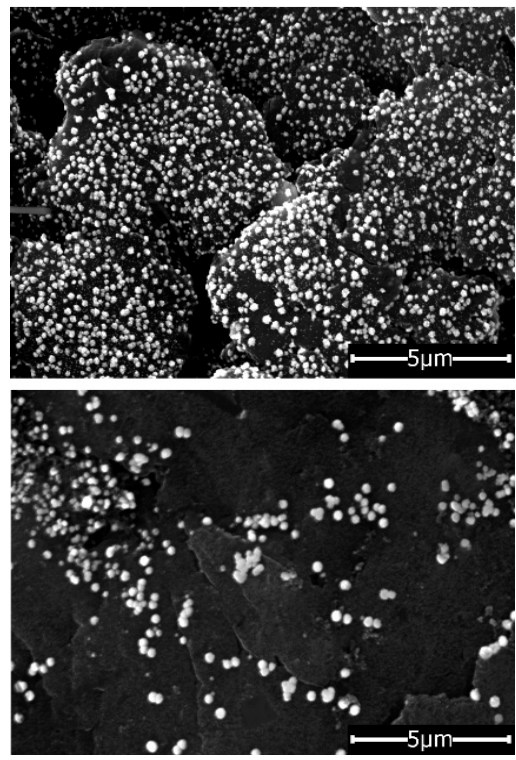

Figure 2. FESEM images of GCE/N-rGO-Au (20 cycles) and GCE/rGO-Au (30 cycles).

To determine the surface elemental composition of nanocomposites, the X-ray photoelectron spectroscopy (XPS) was applied. It was revealed that a $7.6 \%$ at. of Au was electrodeposited on the surface of N-rGO, which was followed by a decrease in the nitrogen content from $6.4 \%$ at. for N-rGO to $2.7 \%$ at. for N-rGO-Au. The lower nitrogen amount on the surface of N-rGO proves that AuNPs anchor on the N-rich regions covering nitrogen atoms, which leads to a decrease of the nitrogen content on the surface of N-rGO-Au determined by XPS.

\section{Conclusions}

This study shows that N-rGO is a promising graphene material to obtain Au-containing nanocomposites with a homogeneous distribution of AuNPs, which result in enhanced working parameters of DA sensors. Despite a lower number of cycles of the electrodeposition, the efficiency of the AuNPs deposition on the graphene nanosheets was higher for N-rGO than for rGO.

Funding: Following research was financially supported by a statutory activity subsidy from the Polish Ministry of Science and Higher Education for the Faculty of Chemistry of Wrocław University of Science and Technology.

Conflicts of Interest: The authors declare no conflict of interest.

\section{References}

1. Jackowska, K.; Krysinski, P. New trends in the electrochemical sensing of dopamine. Anal. Bioanal. Chem. 2013, 405, 3753-3771, doi:10.1007/s00216-012-6578-2.

2. Shao, Y.; Wang, J.; Wu, H.; Liu, J.; Aksay, I.A.; Lin, Y. Graphene Based Electrochemical Sensors and Biosensors: A Review. Electroanalysis 2010, 22, 1027-1036.

3. Xu, H.; Ma, L.; Jin, Z. Nitrogen-doped graphene: Synthesis, characterizations and energy applications. J. Energy Chem. 2018, 27, 146-160.

4. Wang, C.; Du, J.; Wang, H.; Zou, C.; Jiang, F.; Yang, P.; Du, Y. A facile electrochemical sensor based on reduced graphene oxide and Au nanoplates modified glassy carbon electrode for simultaneous detection of ascorbic acid, dopamine and uric acid. Sensors Actuators B Chem. 2014, 204, 302-309. 
5. Luo, X.; Morrin, A.; Killard, A.J.; Smyth, M.R. Application of Nanoparticles in Electrochemical Sensors and Biosensors. Electroanalysis 2006, 18, 319-326.

6. Gryglewicz, S.; Grzyb, B.; Díez, N.; Śliwak, A. Enhanced reduction of graphene oxide by high-pressure hydrothermal treatment. RSC Adv. 2015, 5, 81831-81837.

(C) 2019 by the authors. Licensee MDPI, Basel, Switzerland. This article is an open access article distributed under the terms and conditions of the Creative Commons Attribution (CC BY) license (http://creativecommons.org/licenses/by/4.0/). 